\title{
STRING OF YOUNG CLUSTERS LINKING THE MAGELLANIC CLOUDS
}

\author{
L. GRONDIN, S. DEMERS \\ Université de Montréal \\ Montreal \\ Canada \\ W.E. KUNKEL \\ The Observatories of the Carnegie Institution of Washington \\ La Serena \\ Chile \\ M.J. IRWIN \\ Institute of Astronomy \\ Cambridge University \\ Cambridge \\ England
}

\begin{abstract}
We present deep CCD photometry of three associations lying between the Magellanic Clouds and sitting on the HI ridge. ZAMS fittings show that these clusters are located at the distance of the Clouds. Furthermore, a slight distance gradient is observed. Main sequences matched to isochrones reveal that these aggregates are very young, no more than $10^{8}$ years old. An age difference from east to west is also suggested.
\end{abstract}

The photographic survey by Irwin et al. (1990) of the Magellanic Bridge has revealed that the Wing of the SMC extends eastward, towards the LMC halo. This is underlined by the presence of several aggregates of blue stars distributed along the Bridge, sitting on the HI ridge. We present CCD photometry of three aggregates located between the classical eastern tip of the SMC Wing and the LMC halo. Their coordinates are given in Table 1, as well as their deduced distances from appropriate ZAMS fitting, their reddening and the number of main sequence stars with $V<20$ found within each CCD field. The analysis of the spatial distribution of the main sequence stars reveals that we are dealing with associations of stars, rather than clusters. Associations 5 and 6 do not show obvious central concentration and are rather loose. Although Association 2 is more concentrated, Grondin et al (1990 - GDKI) showed that with a diameter of $\sim 85 \mathrm{pc}$, it is larger than typical SMC clusters by an order of magnitude. We used the ZAMS of the Revised Yale Isochrones (RYI) (Green et al. 1987) to determine the distance of these associations. GDKI argued that the reddening for Association 2 is negligible. A value of $E(B-V)=0.03$ is also adopted for Association 5. Demers et al. (1990) determine for Association 6 that $E(B-V)=0.10$. The abundance of these young stars is unknown. Following GDKI, we adopt $[\mathrm{Fe} / \mathrm{H}]=-0.65$ and select the ZAMS accordingly. The distances found are shown in Table 1.

Our photometry reveals that the stellar bridge between the Clouds shows a distance gradient. Such a result is not unexpected - the bridge starts at the SMC Wing tip and ends in the LMC halo which is closer to us. An age variation, even a gradient, among the observed associations is possible. Our preliminary results cannot be compared to adequate theoretical models. It is not 
possible at the present time to establish accurately the age of the young aggregates. We believe, nevertheless, that they are less than $10^{8}$ years old.

We display below, the CMD of Associations 2 and 6. Superimposed on the data are the youngest isochrones available from RYI, $150 \times 10^{6}$ years with $Y=0.3$ and $Z=0.004$, and the ZAMS from Schmidt-Kaler (1982). The latter is corrected to account for a $[\mathrm{Fe} / \mathrm{H}]=-0.65$, following the method of Vanden Berg and Poll (1989). These curves are distance and reddening shifted. It seems obvious, then, that Association 6, the one within the halo of the LMC, is younger than Association 2, located one degree East of the SMC Wing tip.

The currently favoured scenario to explain the Bridge implies a close encounter of the SMC with the LMC that would trigger star formation in the densest parts of the inter-Clouds region. From what remains of the HI, (McGee and Newton 1986) we see that the gas is much denser on the SMC side. Our results would then confirm that the associations on the west side were formed first.
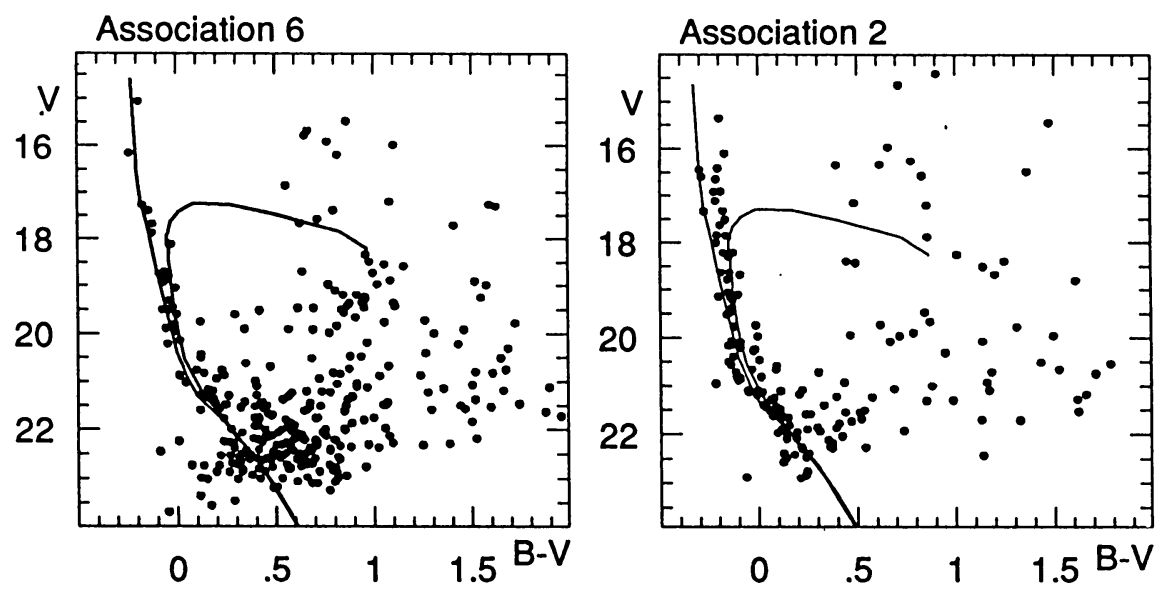

TABLE I.

\begin{tabular}{ccccccc}
\hline \hline Association & $\alpha$ & $\delta$ & $(V-M)$ & $E(B-V)$ & $\begin{array}{c}\text { distance } \\
\text { kpc }\end{array}$ & $\begin{array}{c}\text { number of ms stars } \\
V<20 \mathrm{mag}\end{array}$ \\
\hline 2 & $2^{h} 27^{m}$ & $-73^{\circ} 59^{\prime}$ & $19.05 \pm 0.05$ & 0 & $65 \pm 2$ & 33 \\
5 & $3^{h} 32^{m}$ & $-73^{\circ} 08^{\prime}$ & $18.75 \pm 0.07$ & 0.03 & $54 \pm 2$ & 7 \\
6 & $4^{h} 21^{m}$ & $-74^{\circ} 01^{\prime}$ & $18.70 \pm 0.05$ & 0.10 & $53 \pm 2$ & 22 \\
\hline
\end{tabular}

\section{References}

Demers, S., Grondin, L., Irwin, M.J. and Kunkel, W.E. (1990), in preparation.

Green, E.M., Demarque, P., and King, C.R. (1987), The Revised Yale Isochrones and Luminosity Functions, (Yale University Observatory: New Haven).

Grondin, L., Demers, S., Kunkel, W.E. and Irwin, M.J. (1990), Astron. J. in press.

Irwin, M.J., Demers, S. and Kunkel, W.E. (1990), Astron. J. 99, 191.

McGee, R.X. and Newton, L.M. (1986), Proc. Astron. Soc. Australia. 6, 471.

Schmidt-Kaler, Th. (1982), Landolt-Borstein Neue Series, Group 6, Vol. 2B, K. Shaiffers and

H. Voigt (eds.) (Springer-Verlag: Berlin), p.1.

VandenBerg, D.A. and Poll, H.E. (1989), Astron. J. 98, 1451. 